\title{
Coronary artery bypass grafting with adjunctive endarterectomy: A mandatory procedure in complex revascularizations. current results and postoperative considerations
}

\author{
Marco Russo, Paolo Nardi, Guglielmo Saitto, Emanuele Bovio, Antonio Pellegrino and Giovanni Ruvolo \\ Department of Cardiac Surgery, Tor Vergata University Policlinic, Rome, Italy
}

\section{Editorial}

Patients with diffusive coronary artery disease represent a challenge in the current era cardiac surgery. Even if percutaneous coronary intervention has become the treatment of choice for coronary patients, also in presence of left main pathology or multivessel disease with low Syntax Score, CABG remains the best therapy for patients with diffusive coronary disease with a moderate to high Syntax Score pathology [1]. Moreover, it is well known that a complete revascularization is one of the main achievement of coronary surgery [2-3] to reduce the risk of perioperative myocardial infarction and in-hospital mortality, and it is associated with a of late recurrence of symptoms and MACCE [4]. In this scenario the use of CE seems to be an useful and sometimes a mandatory surgical skill for the treatment of complex cases.

The typical profile of a patient candidate to surgical myocardial revascularitazion has changed in the last decade because of PCI has shown good results and the current international guidelines encurage PCI even in patients affected by multivessel disease in presence of a low Syntax Score [5]. Consequently, surgical patients have usually a more diffusive disease, are more frequently older, diabetic and the achievement of a complete revascularization has become more diffucult. This changing in surgical scenario led to CE being reconsidered as a treatment strategy in selected cases and, thus, the reassessment of the technique, its indications and and evaluation of its outcomes is now necessary.

Coronary endarterectomy was introduced by Baeily in 1957 for the treatment of coronary occlusion and during the years his role in cardiac surgery has remained unclear and results remain debated.

This technique was initially performed with the scope to decrease angina in patients affected by diffusive coronary artery occlusive disease, and just later combined with CABG.

The results of this procedure, both early and long ter mas the graft patency remain not well known and many surgeons still have many doubts regarding the real usufullness of this procedure [6-7].

Operative mortality ranges from $2.0 \%$ to $6.5 \%$ and appears higher as compared with CABG without CE [8-10]. Despite the presence of diffuse coronary artery disease, CABG with endarterectomy on LAD offers favorable long-term survival [11].

The late graft patency rates after CE range from $40 \%$ to $81.5 \%$ :
Schwann et al analyzed the results of 288 operated with CABG/ CE. In this series graft failure studied by angiography, interested $33 \%$ of endarterectomized vessels during a mean follow-up of 400 days; graft failure following CE was not statistically different from nonendarterectomized one [5].

Nishi et al. at the Osaka City General Hospital treated 127 patients with diffuse CAD by CABG/CE between January 1994 and April 2003. At a mid-term follow up (16-22 months) $61 \%$ of patients underwent new coronary angiography and $85 \%$ of vessels treated by CE were still patent [12].

The role of antiplatelet therapy to improve long-term graft patency after coronary surgery is well known in literature. While aspirin administration is in the Class I indication after CABG [13], the benefit of concomitant clopidogrel is a controversial issue. Different studies have shown that double antiplatelet therapy increases saphenous vein graft patency and has an important role to mantain the grafts patent after off-pump bypass surgery or after CABG performed in stead of acute coronary syndrome [14-16]. On the other hand these results are limited to specifical subgroups of patients and the majority of clinical trials have failed to demonstrate an improvement in graft patency with DAPT. Despite that, there are no evidence and no specific protocols regarding postoperative management of patients treated with CABG/ $\mathrm{CE}$ in term of anticoagulation/antiplatelet antiaggregation specific regimen.

We have focused our attention on the role of different medical approaches on patients treated by means of coronary endarterectomy and retrospectively reviewed 90 consecutive patients ( $67 \pm 8.2$ years, 66 men) who underwent isolated CABG with CE between January 2006 and December 2013 at our Institution. Among these, we identified two homogeneous groups treated with aspirin (100 mg daily; Group SAT, $\mathrm{n}=20,66.8 \pm 9.4$ years, $17 \mathrm{men})$ vs aspirin + clopidogrel $(75 \mathrm{mg}$ daily; Group DAT, $\mathrm{n}=52,66.8 \pm 7.8$ years, 37 men) during postoperative period and at follow-up (mean follow-up duration was $71.3 \pm 32.7$, $100 \%$ complete).

Correspondence to: Marco Russo, Cardiac Surgery Division, Tor Vergata University Policlinic, Viale Oxford 81, 00133 Rome, Tel +39 0620903536, Fax +390620903538, E-mail: mar.russo1987@gmail.com

Received: July 25, 2016; Accepted: July 28, 2016; Published: July 31, 2016 
Russo M (2016) Coronary artery bypass grafting with adjunctive endarterectomy: A mandatory procedure in complex revascularizations. current results and postoperative considerations

As far as it concerns the early results the in-hospital operative mortality was $2.7 \%$ (SAT $0 \%$ vs. DAT $3.8 \%$; $\mathrm{p}=0.38$ ) whereas the perioperative myocardial infarction (Troponin I $>20 \mathrm{mg} / \mathrm{dl}$ ) was $12.3 \%$ (SAT $15.0 \%$ vs. DAT 11.5\%; $\mathrm{p}=0.67$ ) and major bleeding requiring surgical re-esploration was $4.1 \%$ (SAT $10.0 \%$ vs. DAT $1.9 \%$; $\mathrm{p}=0.12$ ). Focusing of follow up results, freedom from cardiac death at $3 y, 5 y$ and $7 \mathrm{y}$ was $95 \pm 5 \%, 89 \pm 6 \%, 84 \pm 9 \%$ vs. $88 \pm 4 \%, 88 \pm 4 \%$ and $85 \pm 5 \%$ in Group SAT and DAT respectively while freedom from need of new PCI at $3 y, 5 y$ and $7 y$ was $100 \%, 100 \%$ and, $93 \pm 6 \% v$ s. $100 \%$, in SAT and DAT groups respectively ( $\mathrm{p}=\mathrm{ns})$. During FU two patients needed new PCI: one from group SAT after 60 months and one from group DAT after 90 months. After 90 months there were in group DAT just 9 patients at risk and so we could not consider this data as statistically powerfull. During all the FU period no patient required new surgical revascularization while no differences were observed regarding the need of new PCI. Similar results were obtained regarding freedom from MACCE and symptoms at follow up.

In our report there are no statistically significant differences between the two different antiplatet protocols used even if, considering absoloute terms, the DAT group showed a trend versus better results in all the outcomes studied included.

In the "new era coronary surgery" think about how to treat patients with diffusive coronary disease seems to be mandatory. In our experience $\mathrm{CE}$ is a reasonable option with acceptable mid-term results. Different postoperative antiplatelet or anticoagulation regimen could have a key role in the improving of clinical results and graft patency. No differences were observed in term of major clinical outcomes between patients treated with single vs dual antiplatelet therapy at least in a midterm period of follow-up, even if the double antiplatelet therapy seems to guarantee a better event-free survival.

\section{References}

1. Windecker S, Kolh P, Alfonso F et al. (2014) ESC/EACTS Guidelines on myocardial revascularization: The Task Force on Myocardial Revascularization of the European Society of Cardiology (ESC) and the European Association for Cardio-Thoracic Surgery (EACTS) Developed with the special contribution of the European Association of Percutaneous Cardiovascular Interventions (EAPCI). Eur Heart J 35: 2541-2519.

2. Zimarino M, Ricci F, Romanello M, Di Nicola M, Corazzini A, et al. (2015) Complete myocardial revascularization confers a larger clinical benefit when performed with state-of-the-art techniques in high-risk patients with multivessel coronary artery disease: A meta-analysis of randomized and observational studies. Catheter Cardiovasc Interv.
3. Garcia S, Sandoval Y, Roukoz H, Adabag S, Canoniero M, et al. (2013) Outcomes after complete versus incomplete revascularization of patients with multivessel coronary artery disease: a meta-analysis of 89,883 patients enrolled in randomized clinical trials and observational studies. J Am Coll Cardiol 62:1421-1431.

4. Papakonstantinou NA, Baikoussis NG, Apostolakis E (2014) Coronary endarterectomy: new flavors from old recipes. $J$ Cardiol 63: 397-401. [Crossref]

5. Schwann TA, Zacharias A, Riordan CJ, Durham SJ, Shah AS, et al. (2007) Survival and graft patency after coronary artery bypass grafting with coronary endarterectomy: role of arterial versus vein conduits. Ann Thorac Surg 84: 25-31. [Crossref]

6. Effler DB, Groves LK, Sones FM Jr, Shirey EK (1964) Endarterectomy In The Treatment Of Coronary Artery Disease. J Thorac Cardiovasc Surg 47: 98-108. [Crossref]

7. Livesay JJ, Cooley DA, Hallman GL, Reul GJ, Ott DA, et al. (1986) Early and late results of coronary endarterectomy. Analysis of 3,369 patients. J Thorac Cardiovasc Surg 92: 649-660. [Crossref]

8. Byrne JG, Karavas AN, Gudbjartson T, et al. (2004) Left anterior descending coronary endarterectomy: early and late results in 196 consecutive patients. Ann Thorac Surg 78: $867-873$.

9. Eryilmaz S, Inan MB, Eren NT, Yazicioglu L, CorapcioÄŸlu T, et al. (2003) Coronary endarterectomy with off-pump coronary artery bypass surgery. Ann Thorac Surg 75: 865-869. [Crossref]

10. Vohra HA, Kanwar R, Khan T, Dimitri WR (2006) Early and late outcome after offpump coronary artery bypass graft surgery with coronary endarterectomy: a singlecenter 10-year experience. Ann Thorac Surg 81: 1691-1696. [Crossref]

11. Takahashi M, Gohil S, Tong B, Lento P, Filsoufi F, et al. (2013) Early and mid-term results of off-pump endarterectomy of the left anterior descending artery. Interact Cardiovasc Thorac Surg 16: 301-305. [Crossref]

12. Nishi H, Miyamoto S, Takanashi S, Minamimura H, Ishikawa T, et al. (2005) Optimal method of coronary endarterectomy for diffusely diseased coronary arteries. Ann Thorac Surg 79: 846-852. [Crossref]

13. Patrono C, Bachmann F, Baigent C, Bode C, De Caterina R, et al. (2004) Expert consensus document on the use of antiplatelet agents. The task force on the use of antiplatelet agents in patients with atherosclerotic cardiovascular disease of the European society of cardiology. Eur Heart J 25: 166-181. [Crossref]

14. Deo SV, Dunlay SM, Shah IK, Altarabsheh SE, Erwin PJ, et al. (2013) Dual antiplatelet therapy after coronary artery bypass grafting: is there any benefit? A systematic review and meta-analysis. J Card Surg 28: 109-116. [Crossref]

15. Shapira OM, Akopian G, Hussain A, Adelstein M, Lazar HL, et al. (1999) Improved clinical outcomes in patients undergoing coronary artery bypass grafting with coronary endarterectomy. Ann Thorac Surg 68: 2273-2278. [Crossref]

16. Bomb R, Oliphant CS, Khouzam RN (2015) Dual Antiplatelet Therapy After Coronary Artery Bypass Grafting in the Setting of Acute Coronary Syndrome. Am J Cardiol 116 148-154. [Crossref]

Copyright: (C2016 Russo M. This is an open-access article distributed under the terms of the Creative Commons Attribution License, which permits unrestricted use, distribution, and reproduction in any medium, provided the original author and source are credited. 